\section{(2) OPEN ACCESS}

\title{
CD109 regulates the inflammatory response and is required for the pathogenesis of rheumatoid arthritis
}

\author{
Guanhua Song, ${ }_{1}^{1}$ Tingting Feng, ${ }^{2}$ Ru Zhao, ${ }^{2}$ Qiqi Lu, ${ }^{3}$ Yutao Diao, ${ }^{1}$ Qingwei Guo, ${ }^{4}$ \\ Zhaoxia Wang, ${ }^{1}$ Yuang Zhang, ${ }^{3}$ Luna Ge, Jihong Pan, ${ }^{5}$ Lin Wang (D) , Jinxiang Han $^{5}$
}

\begin{abstract}
Handling editor Josef $S$
Smolen

- Additional material is published online only. To view please visit the journal online (http://dx.doi.org/10. 1136annrheumdis-2019215473)
\end{abstract}

For numbered affiliations see end of article.

\section{Correspondence to}

Dr Lin Wang, Key Laboratory for Rare and Uncommon Diseases of Shandong Province, Research Center for Medicinal

Biotechnology, Shandong First Medical University \& Shandong Academy of Medical Sciences, Jinan 250062, China; wanglin.83@163.com Professor Jinxiang Han; sams-h2016@163.com

Received 2 April 2019 Revised 31 July 2019 Accepted 6 August 2019 Published Online First 27 August 2019
Check for updates

(C) Author(s) (or their employer(s)) 2019. Re-use permitted under CC BY-NC. No commercial re-use. See rights and permissions. Published by BMJ.

To cite: Song G, Feng T, Zhao R, et al. Ann Rheum Dis 2019;78:1632-1641.

\section{ABSTRACT}

Objective The aim of this study was to investigate the role of CD109 in rheumatoid arthritis (RA) fibroblast-like synoviocytes (FLSs) and to evaluate its potential as a therapeutic target.

Methods CD109 expression was examined in synovial tissues and FLSs from RA patients and collagen-induced arthritis (CIA) model mice. CD109deficient mice were developed to evaluate the severity of CIA. Small interfering RNAs and a neutralising antibody against CD109 (anti-CD109) were designed for functional or treatment studies in RA FLSs and CIA. Results CD109 was found to be abundantly expressed in the synovial tissues from RA patients and CIA mice. CD109 expression in RA FLSs was upregulated by inflammatory stimuli, such as interleukin- $1 \beta$ and tumour necrosis factor- $\alpha$ Silencing of CD109 or anti-CD109 treatment reduced proinflammatory factor production, cell migration, invasion, chemoattractive potential and osteoclast differentiation, thereby reducing the deleterious inflammatory response of RA FLSs in vitro. Mice lacking CD109 were protected against arthritis in the CIA model. Anti-CD109 treatment prevented the onset and ameliorated the severity of CIA lesions.

Conclusion Our study uncovers an antiarthritic role for CD109 and suggests that CD109 inhibition might serve as a promising novel therapeutic strategy for RA.

\section{INTRODUCTION}

Rheumatoid arthritis (RA) is a systemic autoimmune disease characterised by chronic inflammation and hyperplasia of fibroblast-like synoviocytes (FLSs). ${ }^{1}$ RA FLSs display an aggressive phenotype and produce excessive amounts of proinflammatory cytokines and matrix-degrading enzymes, resulting in joint dysfunction and destruction. ${ }^{2}$ Hence, identifying key factors that specifically target RA FLS-mediated inflammation may provide novel therapeutic targets for RA.

CD109 is a cell-surface antigen that belongs to the $\alpha 2$-macroglobulin/C3, C4, C5 family of thioester-containing proteins. ${ }^{3}$ As a glycosylphosphatidylinositol-anchored protein, ${ }^{4}$ increased expression of CD109 in various tumours is indicative of a more aggressive phenotype and poor prognosis. ${ }^{5-7}$ Since RA FLSs exhibit tumourlike proliferation and invasion behaviours, ${ }^{2}$ it is possible that CD109 may play a role in the aggressive phenotype of RA FLSs. It has been reported that CD109 interacts with glucose-regulated protein 78 (GRP78), a classical endoplasmic

\section{Key message}

What is already known about this subject?

- CD109 regulates diverse pathogenic processes, including fibrosis, osteoporosis and tumour metastasis.

- CD109 inhibits transforming growth factor (TGF)- $\beta$ signalling by enhancing SMAD7/ Smurf2-dependent degradation of the TGF- $\beta$ receptor.

What does this study add?

- CD109 is required to sustain rheumatoid arthritis (RA) fibroblast-like synoviocyte (FLS)induced inflammation.

- CD109 interacts with and stabilises ENO1 to regulate the RA FLS-mediated inflammatory response.

- An anti-CD109 antibody shows both prophylactic and therapeutic effects in collageninduced arthritis mice.

How might this impact on clinical practice or future developments?

- CD109 could be a promising treatment target for RA.

reticulum (ER) chaperone, to inhibit transforming growth factor (TGF- $\beta$ ) signalling in response to ER stress. ${ }^{8}$ Interestingly, GRP78 acts as a pathological factor that contributes to the severity of RA, ${ }^{9}$ suggesting that CD109 is likely involved in the pathological progression of RA.

A previous study demonstrated that CD109 inhibits TGF- $\beta$ signalling by enhancing SMAD7/ Smurf2-dependent degradation of the TGF- $\beta$ receptor TGF $\beta$ RI. $^{10}{ }^{11}$ More interestingly, although CD109 inactivates TGF- $\beta$ signalling and subsequent fibrosis, fibroblasts with increased CD109 protein levels still produce excessive amounts of extracellular matrix (ECM). ${ }^{12}$ It is thus believed that CD109 is insufficient to completely counteract the effect of TGF- $\beta$ activation on fibrosis, and the exact role of elevated CD109 in fibroblasts remains unclear. Recently, CD109 has been shown to activate Jak-Stat 3 signalling, which consequently leads to a metastatic phenotype in lung cancer cells. ${ }^{13}$ This evidence indicates that CD109 functions in a cell-specific manner, and it would be interesting to characterise the exact role of CD109 in RA FLSs. 

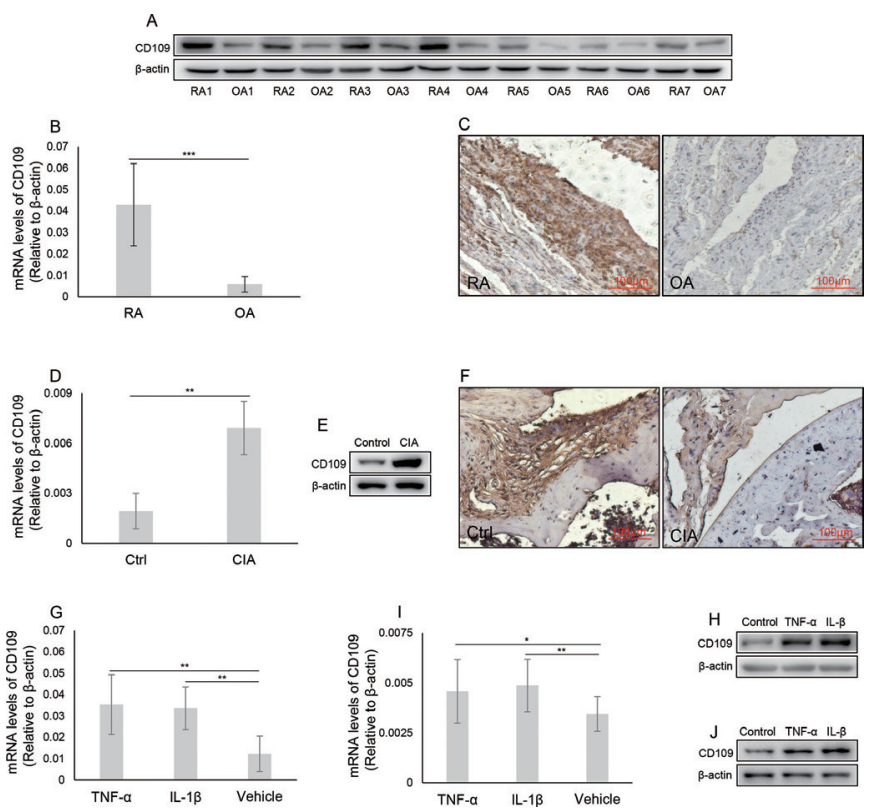

Figure 1 Expression of CD109 in synovial tissues extracted from RA patients and CIA model mice. (A) Western blot analysis of CD109 protein in synovial tissues from RA $(n=7)$ and $O A(n=7)$ patients. $\beta$-Actin was used as a loading control. (B) Quantitative real-time ( $q R T)$ PCR analysis of CD109 mRNA levels in FLSs from RA $(n=7)$ and OA $(n=7)$ patients. ${ }^{* *} p<0.001$. (C) Immunohistochemical staining of RA $(n=16)$ and OA $(n=7)$ synovial tissues using antibodies against CD109. Original magnification is 200x. FLSs were collected from DBA/1 J mice immunised with collagen (CIA mice) or bovine serum albumin (BSA, referred to as Ctrl mice). qRT-PCR and Western blot assays were performed to assess the levels of CD109 mRNA (D) and protein (E). $\mathrm{n}=3$ per group, ${ }^{* *} \mathrm{p}<0.01$. (F) Immunohistochemical staining of CD109 from the hind paw in CIA and Ctrl mice. FLSs from RA patients ( $G$ and $\mathrm{H}$ ) or CIA model mice (I and J) were stimulated with TNF- $\alpha$ or IL-1 $\beta$ for 24 hours. Total RNA and protein levels were subjected to qRT-PCR and Western blotting for the analysis of CD109 mRNA ( $G$ and I) and protein ( $\mathrm{H}$ and J) levels. qRT-PCR data in $\mathrm{G}$ and I are expressed as the mean of six samples (three male and three female) or three mice from two independent experiments. Western blot data in $\mathrm{H}$ and $\mathrm{J}$ represent two independent experiments from six samples (three males and three females) or three mice with similar results. ${ }^{*} p<0.05$ and ${ }^{* *} p<0.01$ compared with vehicle. CIA, collagen-induced arthritis; FLSs, fibroblast-like synoviocytes; IL-1 $\beta$, interleukin-1 $\beta$; OA, osteoarthritis; RA, rheumatoid arthritis; TNF- $\alpha$, tumour necrosis factor- $\alpha$.

The present study was conducted to investigate the role of CD109 in RA FLSs and collagen-induced arthritis (CIA) models and to assess the potential of CD109 inhibition as a treatment approach in RA.

\section{MATERIALS AND METHODS}

Detailed methods and sequence primers are described in the online supplementary materials and methods.

\section{Reagent and cell stimulation}

RA FLSs were plated in 24 -well plates $\left(3-5 \times 10^{5}\right.$ cells/well) in Dulbecco's Modified Eagle's Medium and stimulated for the indicated time points with the following agents: tumour necrosis factor (TNF)- $\alpha$ (10 ng/mL, Abnova, Taiwan, China), interleukin (IL)-1 $\beta$ (10 ng/mL, Abnova), cycloheximide (CHX, $10 \mu \mathrm{g} / \mathrm{mL}$, Calbiochem), galunisertib (10 $\mu \mathrm{M}$, Selleck), TGF- $\beta 1$ ( $1 \mathrm{ng} / \mathrm{mL}$ or $10 \mathrm{ng} / \mathrm{mL}, \mathrm{R} \& \mathrm{D})$, macrophage colony-stimulating factor (M-CSF; $30 \mathrm{ng} / \mathrm{mL}, \mathrm{R} \& \mathrm{D})$ and RANKL $(50 \mathrm{ng} / \mathrm{mL}$, $\mathrm{R} \& \mathrm{D})$. The dilution buffer, phosphate buffer saline, was applied as vehicle control.

\section{RESULTS}

\section{CD109 is abundantly expressed in the synovial tissues of} patients with RA

CD109 was first found to be abundantly expressed in all RA patient synovial tissues but dramatically reduced in osteoarthritis (OA) samples (figure 1A). In vitro, CD109 mRNA expression in RA FLSs was relatively higher than that in OA FLSs (figure 1B). Immunohistochemical staining showed that CD109 was expressed at higher levels in RA synovial tissues than in OA synovial tissues and mainly distributed in the lining layer of the RA synovial tissues (figure 1C). A similar increase in CD109 expression was also observed in FLSs (figure 1D,E) and synovial tissues (figure 1F) from CIA mice when compared with those from wild-type (WT) mice. In addition, CD109 expression was elevated at the mRNA (figure $1 \mathrm{G}, \mathrm{I}$ ) and protein (figure $1 \mathrm{H}, \mathrm{J}$ ) levels in FLSs from RA patients and CIA mice following TNF- $\alpha$ and IL-1 $\beta$ stimulation, but not following IL-1 $\alpha$, IL- 6 and IL-17 stimulation (online supplementary figure 1$){ }^{14}$

\section{Effects of CD109 on the inflammatory phenotype of RA FLSs}

RA FLSs can secrete IL-6, IL-8, matrix metalloproteinase (MMP)-1, MMP-3 and MMP-13 in response to IL-1 $\beta$ and TNF- $\alpha$ stimulation, and these factors are implicated in the RA FLS-mediated inflammatory response. ${ }^{15-22}$ We next measured the levels of these proteins in the supernatant of siCD109. transfected or siCtrl-transfected RA FLS. The silencing efficiency of small interfering RNAs (siRNAs) against CD109 was confirmed by Western blot (figure $2 \mathrm{~A}$ ). The data showed that silencing of CD109 significantly reduced the levels of IL-6, IL-8, MMP-1 and MMP-3 (figure 2B-E), but not MMP-13 (data not shown), in the supernatants of RA FLSs with or without TNF- $\alpha$ and IL-1 $\beta$ stimulation. Additionally, CD109 knockdown resulted in an obvious reduction in TNF- $\alpha$-induced ${ }^{23} 24$ or IL-1 $\beta$-induced ${ }^{25-27}$ phosphorylation of AKT serine/threonine kinase (Akt), nuclear factor-kappa B (NF- $\kappa \mathrm{B})$, signal transducer and activator of transcription 3 (Stat3) and p38 MAPK signalling proteins (figure 2F). CXCL9/10 modulates RA immune responses by activating and recruiting leucocytes. ${ }^{28} 29$ CD109 knockdown significantly reduced the production of CXCL9 and CXCL10 (figure 2G,H) in RA FLSs. The number of migrating leucocytes cultured with conditioned medium (CM) from siCD109-transfected RA FLSs also decreased significantly (figure 2I), suggesting decreased chemoattractive potential of RA FLSs. Furthermore, RA FLSs with CD109 downregulation displayed reduced levels of migration (figure 2J) and invasion (figure $2 \mathrm{~K}$ ). However, proliferation and apoptosis did not differ between siCD109-transfected RA FLSs and siCtrl-transfected RA FLSs (online supplementary figure 2A-B). Together, these data indicate the importance of CD109 inhibition in limiting the RA FLS-mediated inflammatory response.

CD109 acts as a negative regulator of TGF- $\beta$ signalling by promoting the degradation of its receptor TGF $\beta$ RI. ${ }^{10-12}$ However, CD109 is insensitive to TGF- $\beta 1$ in RA FLSs (online supplementary figure $3 \mathrm{~A}$ ). Blocking TGF- $\beta$ signalling by treating cells with the specific inhibitor, galunisertib, did not attenuate the CD109 overexpression-induced increase in IL-6, IL-8, MMP-1, MMP-3, CXCL9 and CXCL10 expression in RA FLSs (online supplementary figure 3B-G). Our data suggest that CD109 regulates the RA FLS-mediated inflammation in a TGF- $\beta$ signallingindependent manner. 

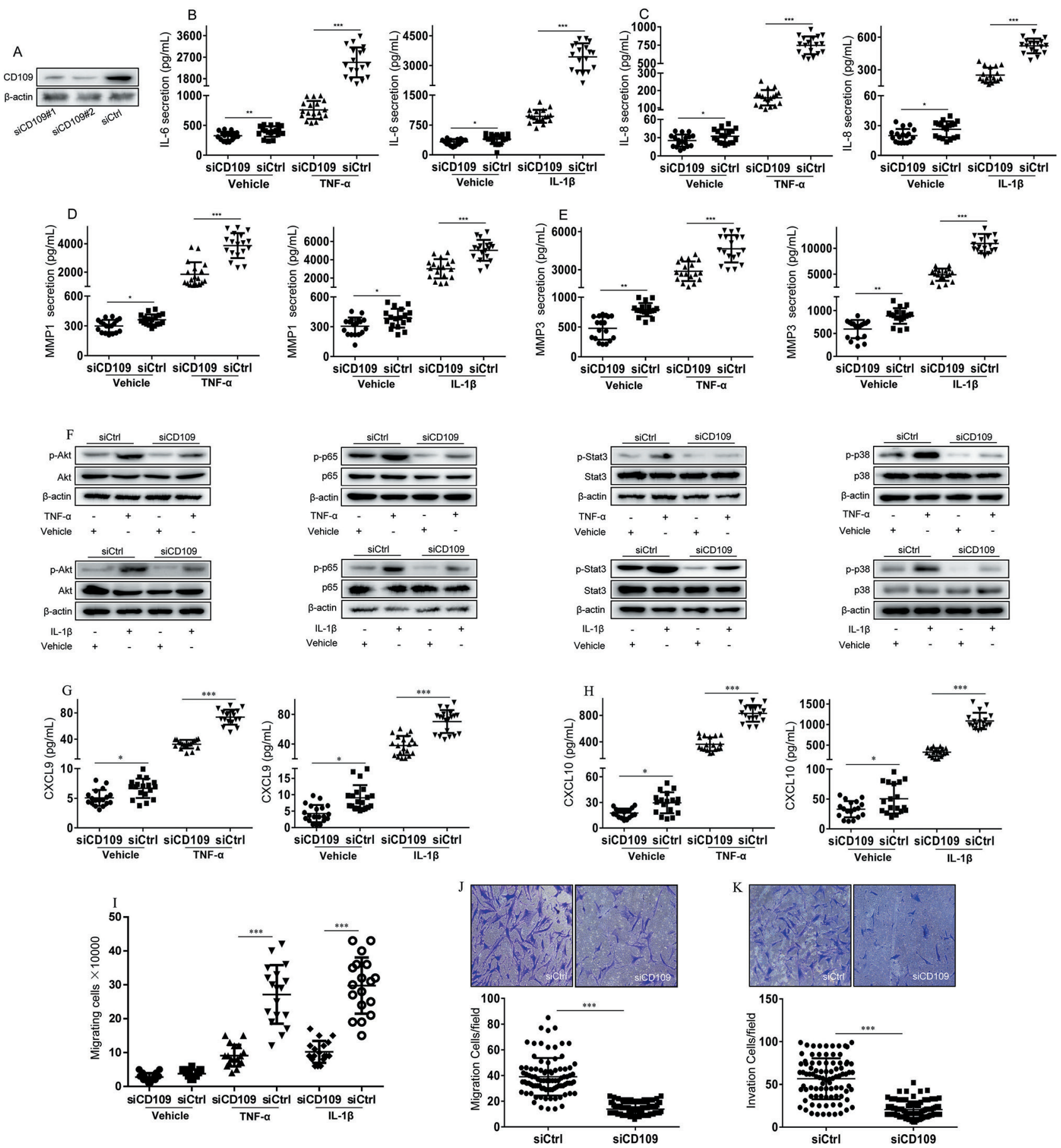

Figure 2 Effects of CD109 silencing on FLS in RA. (A) Silencing efficiency of siRNA targeting CD109 (siCD109) was detected by Western blot. The two siRNAs were combined at equal concentrations for the subsequent experiments. The negative control siRNA is referred to as siCtrl. The results are representative of two independent experiments with three different samples in each. (B-E) Following transfection with siCD109 or siCtrl for 12 hours, RA FLSs were stimulated with TNF- $\alpha$ and IL-1 $\beta$ for another 24 hours. IL-6 (B), IL-8 (C), MMP-1 (D) and MMP-3 (E) levels in the supernatant were analysed by ELISA. (F) Using similar treatments as B-E, total and phosphorylated levels of Akt, p65, Stat3 and p38 MAPK were analysed by Western blot. Western blot data are representative of two independent experiments from six different patients (three males and three females) with similar results. (G and H) RA FLSs were transfected with siCD109/siCtrl for 12 hours, followed by TNF- $\alpha$ or IL-1 $\beta$ stimulation for another 24 hours. CXCL9 (G) and CXCL10 (H) levels in the culture supernatant were measured by ELISA. Data in B-E, G and H are expressed as the mean of six samples (three males and three females) $\pm S D$ and represent three independent experiments. (I) After treatment as indicated in $B-E$, cell-free RA FLSs supernatant was collected and used as a chemotactic source for healthy donor peripheral blood leucocytes $(n=3)$ in a transwell migration system for 6 hours. The number of migrating leucocytes in the lower compartment was counted. The migration (J) and invasion (K) abilities were assessed in siCD109-transfected or siCtrl-transfected RA FLS from six different patients (three males and three females). Data represent independent experiments performed in triplicate, and five different fields were selected for cell counting (graph below). RA FLSs passing through the polycarbonate membrane with ECM coating show that cell invasion requires the ECM proteolysis step in addition to migration. ${ }^{*} p<0.05,{ }^{* *} p<0.01$ and ${ }^{* * *} p<0.001$ compared with siCtrl. ECM, extracellular matrix; FLSs, fibroblast-like synoviocytes; IL, interleukin; MMP, matrix metalloproteinase; RA, rheumatoid arthritis; siRNAs, small interfering RNAs; TNF- $\alpha$, tumor necrosis factor- $\alpha$. 

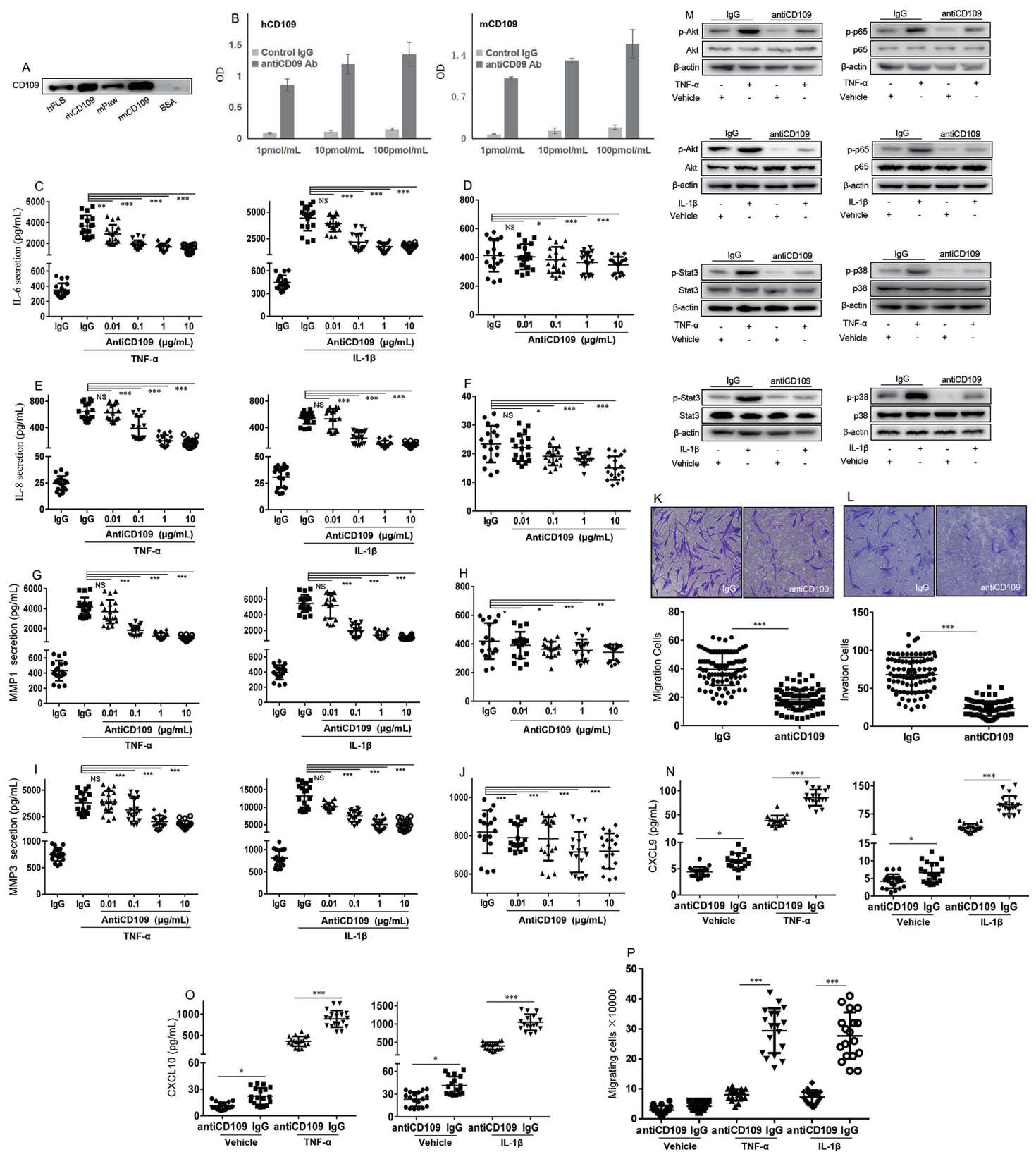

Figure 3 Effects of an antibody against CD109 (anti-CD109) on RA FLSs. (A) Western blot analysis of anti-CD109 against human RA FLS (hFLS), recombinant human CD109 protein (rhCD109), mouse paw (mPaw), recombinant mouse CD109 (rmCD109) and BSA. Data are representative of three independent experiments. (B) ELISA analysis for the binding affinity of anti-CD109 towards human CD109 $(\mathrm{hCD} 109,1 \mu \mathrm{g})$ and mouse CD109 (mCD109, $1 \mu \mathrm{g})$ proteins at the indicated concentration. Anti-CD109 bound to both human and mouse recombinant CD109 protein dose dependently. The data are expressed as the mean \pm SD from three independent experiments. (C-J) Following stimulation with TNF- $\alpha$ and IL-1 $\beta$ or nothing for 12 hours, RA FLSs were subsequently treated with anti-CD109 at the indicated concentration or $\mathrm{lgG}(10 \mu \mathrm{g} / \mathrm{mL})$ for another 24 hours. IL-6 (C and D), IL-8 (E and F), MMP-1 (G and H) and MMP-3 (I and J) levels in the culture supernatant were measured by ELISA. The data are expressed as the mean $\pm S D$ of six samples (three male and three female) and are representative of three independent experiments. ${ }^{*} p<0.05,{ }^{*}{ }^{*}<<0.01$ and ${ }^{* * *} p<0.001$ compared with $\lg G$ in the presence $(C, E, G$ and I) or absence $(D, F, H$ and J) of inflammatory stimuli. The migration (K) and invasion (L) of RA FLSs from six different patients (three males and three females) were assessed and quantified (graph below) after the addition of anti-CD109 $(10 \mu \mathrm{g} / \mathrm{mL})$ or $\mathrm{lgG}(10 \mu \mathrm{g} / \mathrm{mL})$ and incubation for 24 hours. ${ }^{* * *} \mathrm{p}<0.001$ compared with $\mathrm{lgG}$. (M) After exposure to TNF- $\alpha$ or IL-1 $\beta$ for 12 hours, RA FLSs were treated with anti-CD109 $(10 \mu \mathrm{g} / \mathrm{mL})$ for another 24 hours. Total and phosphorylated levels of Akt, p65, Stat3 and p38 MAPK in RA FLSs from six samples (three males and three females) were analysed by Western blot. Western blot data are representative of two independent experiments. RA FLSs were incubated with anti-CD109//gG $(10 \mu \mathrm{g} / \mathrm{mL})$ for 12 hours and treated with TNF- $\alpha$ or IL-1 $\beta$ for another 24 hours. CXCL9 (N) and CXCL10 (0) levels in the culture supernatant were measured by ELISA. Data are expressed as the mean $\pm S D$ of six samples (three males and three females) and are representative of three independent experiments. ${ }^{*} p<0.05$ and ${ }^{* * *} p<0.001$ compared with siCtrl or lgG. (P) After pretreatment with antiCD109/lgG (10 $\mu \mathrm{g} / \mathrm{mL})$ for 12 hours, RA FLSs from six different patients (three males and three females) were treated with inflammatory stimuli for another 24 hours. Cell-free supernatants were collected and used as a chemoattractant source for healthy donor peripheral blood leucocytes $(n=3)$ in a transwell migration system for 6 hours. The number of migrating leucocytes in the lower compartment was counted. ${ }^{* *} p<0.001$ compared with IgG. BSA, bovine serum albumin; FLSs, fibroblast-like synoviocytes; IL, interleukin; IgG, immunoglobulin G; MMP, matrix metalloproteinase; NS, not significant; RA, rheumatoid arthritis; TNF- $\alpha$, tumour necrosis factor- $\alpha$. 


\section{Anti-CD109 treatment ameliorates the arthritis phenotype of RA FLSs}

We next produced an anti-CD109 antibody (anti-CD109) and confirmed its specificity towards human and mouse CD109 by Western blot (figure 3A) and ELISA (figure 3B). The data showed that anti-CD109 treatment dose-dependently reduced the levels of IL-6 (figure 3C,D), IL-8 (figure 3E,F), MMP-1 (figure 3G,H) and MMP-3 (figure 3I,J) irrespective of TNF- $\alpha$ or IL-1 $\beta$ stimulation. Furthermore, anti-CD109 treatment reduced the migration (figure $3 \mathrm{~K}$ ) and invasion (figure $3 \mathrm{~L}$ ) of RA FLSs. However, no obvious differences in the proliferation and apoptosis rates of RA FLSs were detected between anti-CD109 and IgG treatments (online supplementary figure 2C, D). In addition, the levels of phosphorylated Akt, NF- $\mathrm{BB}$, Stat3 and p38 MAPK in TNF- $\alpha$-stimulated or IL-1 $\beta$-stimulated RA FLSs were all reduced by anti-CD109 treatment (figure 3M). Similarly, there was a strong reduction in CXCL9 and CXCL10 levels (figure 3N,O) in RA FLSs and migrating leucocytes (figure $3 \mathrm{P}$ ) cultured with CM from anti-CD109-treated RA FLSs.

In addition, we analysed the effects of anti-CD109 on the TNF- $\alpha$ or IL-1 $\beta$ response in FLSs from CD109-knockout (CD109 KO) and WT mice. Anti-CD109 addition did not reduce the TNF- $\alpha$-induced or IL-1 $\beta$-induced increase in IL-6, MMP-3, CXCL9 and CXCL10 levels in CD109 KO FLSs, as it did in WT FLSs (online supplementary figure 4A-D). These results indicate the engagement of CD109 for anti-CD109.

\section{CD109 interacts with and requires EN01 to regulate the RA FLS-mediated inflammatory response}

To explore the molecular mechanism of CD109 in RA FLSs, glutathione-S-transferase (GST) pull-down assay and mass spectrometry were used. The majority of the identified CD109interacting partners are involved in processes such as metabolism, signalling pathways, protein processing and modification, and cell cycle and apoptosis (online supplementary table 2). ENO1 was selected for further investigation as it is also localised on the cell surface and regulates cytokine production and apoptotic resistance in RA FLSs. ${ }^{30-32}$ Co-immunoprecipitation (figure 4A) and immunofluorescence (figure 4B) analyses confirmed the specific association between CD109 and ENO1 on the surface of RA FLSs; the interaction was enhanced by TNF- $\alpha$ or IL- $1 \beta$ treatment.

Then, we detected the intrinsic relationship between CD109 and ENO1 in RA FLSs. The overexpression or silencing efficiency of CD109 and ENO1 was first confirmed, as shown, respectively, in figure $4 \mathrm{C}$ and $\mathrm{D}$. The overexpression of CD109 increased the level of ENO1 protein (figure 4E) as well as the proportion of ENO1 on the cell surface (figure 4F), irrespective of IL- $1 \beta$ or TNF- $\alpha$ stimulation. In contrast, CD109 silencing or anti-CD109 treatment caused a significant reduction in ENO1 expression (figure 4G) and surface localisation (figure $4 \mathrm{H}, \mathrm{I}$ ). $\mathrm{CHX}$ chase assay results demonstrated that the degradation rate of ENO1 protein increased following CD109 inhibition with either siCD109 or anti-CD109 (figure 4J,K). Furthermore, we observed a marked increase in the levels of inflammatory factors (figure 4L-Q, IL-6, IL-8, MMP-1, MMP-9, CXCL9 and CXCL10), migration (figure 4R), invasion (figure 4S) and phosphorylation of proteins in the proinflammatory pathway (figure 4T) in CD109-overexpressing FLSs compared with control FLSs. However, ENO1 knockdown prevented these effects. These results suggest that ENO1 is required for CD109-mediated regulation of the FLS inflammatory response.

\section{Reduced arthritis severity in CD109-deficient mice}

To further demonstrate the function of CD109 in RA, CIA was induced in WT and CD109 KO mice. When endogenous CD109 was absent (figure 5A), the arthritis score and paw swelling were significantly ameliorated in CD109 KO mice compared with WT mice (figure 5B,C). Likewise, CD109 KO mice also developed a limited degree of inflammatory cell infiltration (granulocytes and $\mathrm{T}$ lymphocytes), synovial hyperplasia, cartilage degradation and bone destruction (figure 5D,E and online supplementary figure 5A-D), accompanied by a decrease in TNF- $\alpha$, IL-1 $\beta$, IL- 6 and IL- 8 levels (figure $5 \mathrm{~F}$ ) in the paws. Inflammatory bone erosion and destruction are known features of RA. ${ }^{33}$ Thus, we examined the effects of global CD109 deletion on bone destruction in CIA models by microcomputed tomography (micro-CT) analysis (figure 5G). The distal tibias from CD109 KO CIA mice displayed an obvious increase in bone volume/tissue volume (BV/TV), total body bone mineral density ( $\mathrm{Tb} \mathrm{BMD})$, trabecular thickness ( $\mathrm{Tb} \mathrm{Th}$ ) and trabecular number $(\mathrm{Tb} \mathrm{N})$ but a decrease in trabecular spacing $(\mathrm{Tb}$ $\mathrm{Sp}$ ) compared with those from the WT counterparts (figure $5 \mathrm{H}$ ). Further evidence showed that bone marrow mononuclear cells (BMMCs) isolated from CD109 KO mice differentiated into osteoclasts in vitro less efficiently than those isolated from WT mice. Notably, this difference was increased between the CD109 KO and WT groups with CIA induction (online supplementary figure $6 \mathrm{~A})$. The receptor activator of NF- $\mathrm{KB}$ ligand (RANKL) promotes osteoclast differentiation, while osteoprotegerin (OPG) attenuates osteoclastic bone destruction, and the OPG/RANKL ratio is critical for RA-induced bone destruction. ${ }^{34}$ Serum from CIA mice was then examined by ELISA, which showed that the serum level of RANKL decreased while that of OPG remained unchanged. This imbalance finally led to an increase in the OPG/RANKL ratio but fewer osteoclasts in CD109 KO mice than WT mice (online supplementary figure 6B-D).

We also investigated whether CD109 regulates RANKL and OPG in FLS. CD109 ablation reduced the TNF- $\alpha$ and IL- $1 \beta$ stimulationinduced increase in RANKL expression in FLS. As OPG levels remained unaffected, the OPG/RANKL ratio increased in CD109 KO FLSs compared with WT FLSs (online supplementary figure $6 \mathrm{E}-\mathrm{H})$. Subsequently, mouse BMMCs were cultured with CM from IL-1 $\beta$-activated or TNF- $\alpha$-activated FLSs and stimulated with M-CSF. CD109 KO FLSs were less efficient at inducing tartrateresistant acid phosphatase-positive multinucleated cells than WT cells (online supplementary figure S6I). Collectively, these findings indicate that ablation of the CD109 gene sufficiently blocks the progression of experimental arthritis.

\section{Effects of prophylactic anti-CD109 treatment}

To evaluate the efficacy of anti-CD109 in the amelioration of CIA, the safety was first analysed. Neither physical and behavioural manifestations nor peripheral blood cell numbers appeared to be significantly affected (online supplementary figure 7). To mimic prophylactic intervention in human RA patients, anti-CD109 was administered on the day of first immunisation with type II collagen. Anti-CD109 treatment dose dependently reduced the arthritis score (figure 6A), hind paw thickness (figure 6B) and swelling (figure 6C) in CIA models. Histological analysis revealed reduced inflammatory cell infiltration (granulocytes and $\mathrm{T}$ lymphocytes), synovial hyperplasia, cartilage degradation and bone destruction in anti-CD109-treated mice (figure 6D,E and online supplementary figure $5 \mathrm{E}-\mathrm{H}$ ). The micro-CT showed that compared with immunoglobulin (Ig) $G$ treatment, antiCD109 treatment greatly reduced bone destruction (figure 6F), as quantitatively evidenced by the increased degree of Tb BMD, 

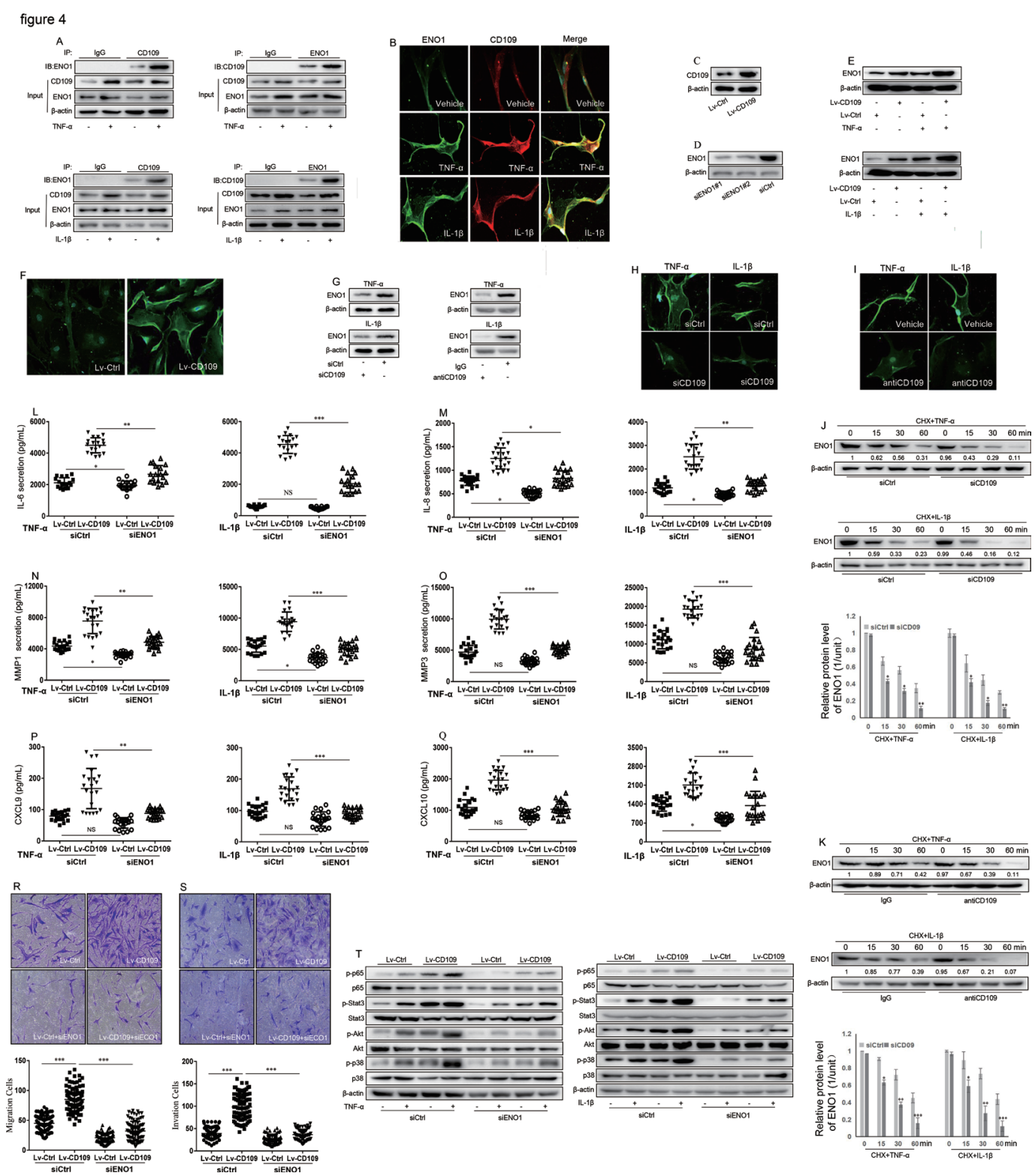

Figure 4 CD109 interacts with and stabilises EN01. (A) RA FLSs were stimulated with TNF- $\alpha$ (upper) or IL-1 $\beta$ (lower) for 24 hours.

Immunoprecipitation and immunoblotting were performed on total protein with anti-CD109 or anti-EN01 antibodies. The results are representative of four samples (two males and two females) from independent experiment. (B) After stimulation with TNF- $\alpha$ or IL-1 $\beta$ for 24 hours, ENO1 (green) and CD109 (red) in RA FLSs were detected by immunofluorescence. (C) Western blot analysis of CD109 protein in RA FLSs following transfection of a lentiviral vector expressing CD109 (Lv-CD109) or its control (Lv-Ctrl). (D) Silencing efficiency of siEN01 was detected by Western blot. The two siRNAs were combined at equal concentrations for the subsequent experiments. Data in $C$ and $D$ are representative of two independent experiments with three different samples. (E) After Lv-CD109 or Lv-Ctrl transfection, RA FLSs were exposed to TNF- $\alpha$ or IL-1 $\beta$ for 24 hours, and EN01 protein levels were measured by Western blot. (F) Surface EN01 was detected by immunofluorescence in Lv-CD109 or Lv-Ctrl-transfected RA FLSs. (G) After transfection of siCD109/siCtrl or treatment with anti-CD109/lgG for 24 hours, the EN01 protein in TNF- $\alpha$-activated or IL-1 $\beta$-activated RA FLSs was measured by Western blot. (H and I) TNF- $\alpha$-stimulated or IL-1 $\beta$-stimulated RA FLSs were transfected with siCD109/siCtrl or treated with anti-CD109/lgG for 24 hours. ENO1 (green) in RA FLS was detected by immunofluorescence. ( $\mathrm{J}$ and K) In the presence of TNF- $\alpha$ or IL-1 $\beta$, RA FLSs were exposed to CHX for different time periods, and the total protein was subjected to Western blotting to analyse ENO1 expression. Band intensities were measured using ImageJ software and are presented as fold change comparisons as indicated. ${ }^{*} p<0.05,{ }^{* *} p<0.01$ and ${ }^{* * *} p<0.001$ compared with siCtrl. CD109overexpressing RA FLSs were transfected with siEN01 or siCtrl for 12 hours and subsequently stimulated with TNF- $\alpha$ and IL-1 $\beta$ for another 24 hours. IL-6 (L), IL-8 (M), MMP-1 (N), MMP-3 (0), CXCL9 (P) and CXCL10 (Q) levels in the culture supernatant were measured by ELISA. Data are expressed as the mean \pm SD of six samples (three males and three females) and are representative of three independent experiments. ${ }^{*} p<0.05,{ }^{* *} p<0.01$ and $* * * p<0.001$ compared with Lv-Ctrl or Lv-CD109 in the siCtrl-transfected group. CD109-overexpressing RA FLSs were transfected with siEN01 or siCtrl for 24 hours. The migration (R) and invasion (S) abilities were assessed and counted (graph below) for RA FLSs from six different patients (three males and three females) and performed in triplicate. Five different fields were selected for cell counting. ${ }^{* *} p<0.001$ compared with Lv-Ctrl. (T) CD109-overexpressing RA FLSs were transfected with siEN01 or siCtrl for 24 hours. Total and phosphorylated levels of Akt, p65, Stat3 and p38 MAPK were analysed by Western blot. Western blot data in $A, E, G, J, K$ and $T$, and immunofluorescence data in $B, F, H$ and $I$ are representative of two independent experiments from six samples (three males and three females) with similar results. CHX, cycloheximide; FLSs, fibroblast-like synoviocytes; IL, interleukin; NS, not significant; RA, rheumatoid arthritis; siRNAs, small interfering RNAs; TNF- $\alpha$, tumor necrosis factor- $\alpha$. 

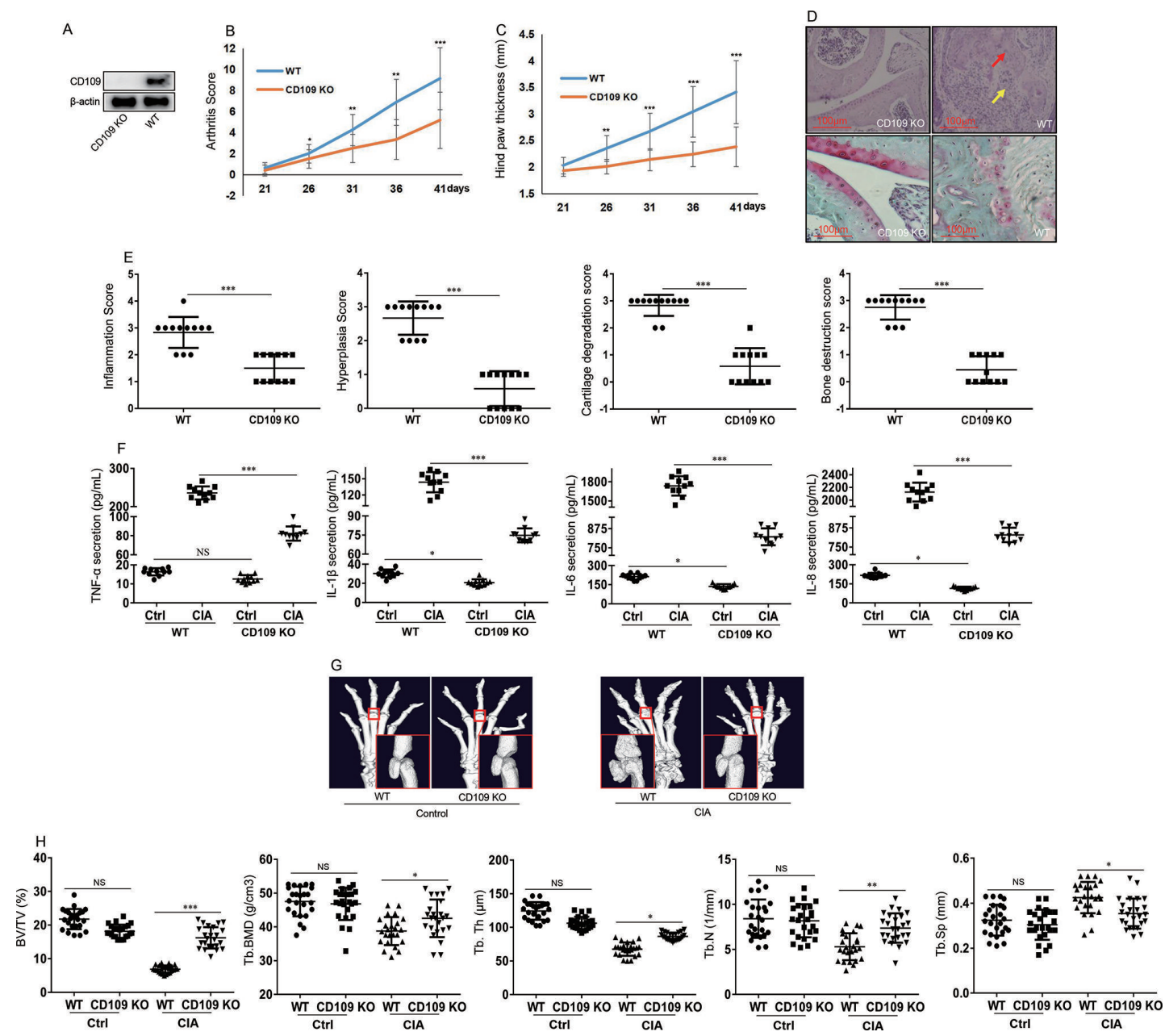

Figure 5 CD109 knockout (CD109 KO) prevents joint destruction in CIA mice. (A) The hind paws from CD109 KO and WT CIA mice at the resolution phase of arthritis were homogenised in cell lysis buffer for Western blot detection of CD109 ( $n=3$ mice per group). (B, C) Arthritis in CD109 KO and WT mice was induced by bovine type II collagen injection ( $n=6$ mice per group and per time point). After the second immunisation, the arthritis score (B) and hind paw thickness (C) were evaluated every 5 days. This experiment is representative of four independent experiments with similar results. ${ }^{*} p<0.05,{ }^{* *} p<0.01$ and ${ }^{* *} p<0.001$ compared with WT. (D) Representative images of haematoxylin-eosin (H\&E, upper) and Safranin $0 /$ Fast green staining (lower) of the interphalangeal joint from CD109 KO and WT CIA mice on the 42nd day after the first immunisation. Pathological changes, including synovial proliferation (yellow arrowhead) and joint destruction (red arrowhead), are shown. (E) Inflammation, hyperplasia, cartilage degradation and bone destruction were evaluated using a scoring system ( $n=12$ per group). ${ }^{* * *} p<0.001$ compared with WT. (F) The hind paws from WT and CD109 KO CIA mice ( $n=6$ mice per group) were homogenised in phosphate-buffered saline (PBS) for ELISA detection of TNF- $\alpha$, IL-1 $\beta$, IL-6 and IL-8 on the 42nd day after the first immunisation. The data are representative of two independent experiments from six mice per group. ${ }^{*} p<0.05$ and ${ }^{* * *} p<0.001$ compared with Ctrl or CIA in the WT group. (G) Representative micro-CT images of hind paws and interphalangeal joints (red square) on the 42nd day after the first immunisation ( $\mathrm{n}=6$ mice per group). (H) Bone volume fraction (BV/TV), trabecular bone mineral density (Tb $\mathrm{BMD}$ ), trabecular thickness ( $\mathrm{Tb} \mathrm{Th}$ ), trabecular number ( $\mathrm{Tb} \mathrm{N}$ ) and trabecular separation (Tb Sp) in the distal tibia were assayed by micro-CT and 3D reconstruction. The data represent four independent experiments with similar results. ${ }^{*} \mathrm{p}<0.05,{ }^{* *} \mathrm{p}<0.01$ and ${ }^{* * *} \mathrm{p}<0.001$ compared with WT. BMD, bone mineral density; CIA, collagen-induced arthritis; KO, knockout; NS, not significant; TNF- $\alpha$, tumour necrosis factor- $\alpha$; WT, wild type.

$\mathrm{BV} / \mathrm{TV}, \mathrm{Tb} \mathrm{Th}$ and $\mathrm{Tb} \mathrm{N}$ but a lower degree of $\mathrm{Tb} \mathrm{Sp}$ in the distal tibias (figure 6G). Furthermore, anti-CD109 treatment effectively decreased the serum level of RANKL but had a minimal effect on the OPG level, thereby increasing the OPG/RANKL ratio and curtailing osteoclast numbers (online supplementary figure $8 \mathrm{~A}-\mathrm{C}$ ) in CIA models.

\section{Effects of therapeutic treatment with anti-CD109}

We also analysed the therapeutic effects of anti-CD109. Compared with IgG-treated CIA model mice, anti-CD109treated model mice showed dose-dependent amelioration with a marked decrease in arthritis score (figure $6 \mathrm{H}$ ), thickness (figure 6I) and hind paw swelling (figure 6J). Histological analysis of affected joints from anti-CD109-treated mice revealed reduced evidence of inflammation (granulocytes and T lymphocytes), synovial hyperplasia, cartilage degradation and bone destruction (figure $6 \mathrm{~K}, \mathrm{~L}$ and online supplementary figure 5I-L). In addition, anti-CD109-treated CIA mice displayed reduced levels of bone erosion and destruction (figure $6 \mathrm{M}, \mathrm{N}$ ), as evidenced by the increase in $\mathrm{BV} / \mathrm{TV}, \mathrm{Tb}$ $\mathrm{BMD}, \mathrm{Tb} \mathrm{Th}$ and $\mathrm{Tb} \mathrm{N}$ but decrease in $\mathrm{Tb} \mathrm{Sp}$ in the distal tibia. Additionally, anti-CD109 treatment decreased the RANKL level, increased the OPG/RANKL ratio and led to a decline in the number of osteoclasts (online supplementary figure 8D-F). Therefore, anti-CD109 treatment alleviates RA progression in vivo. 

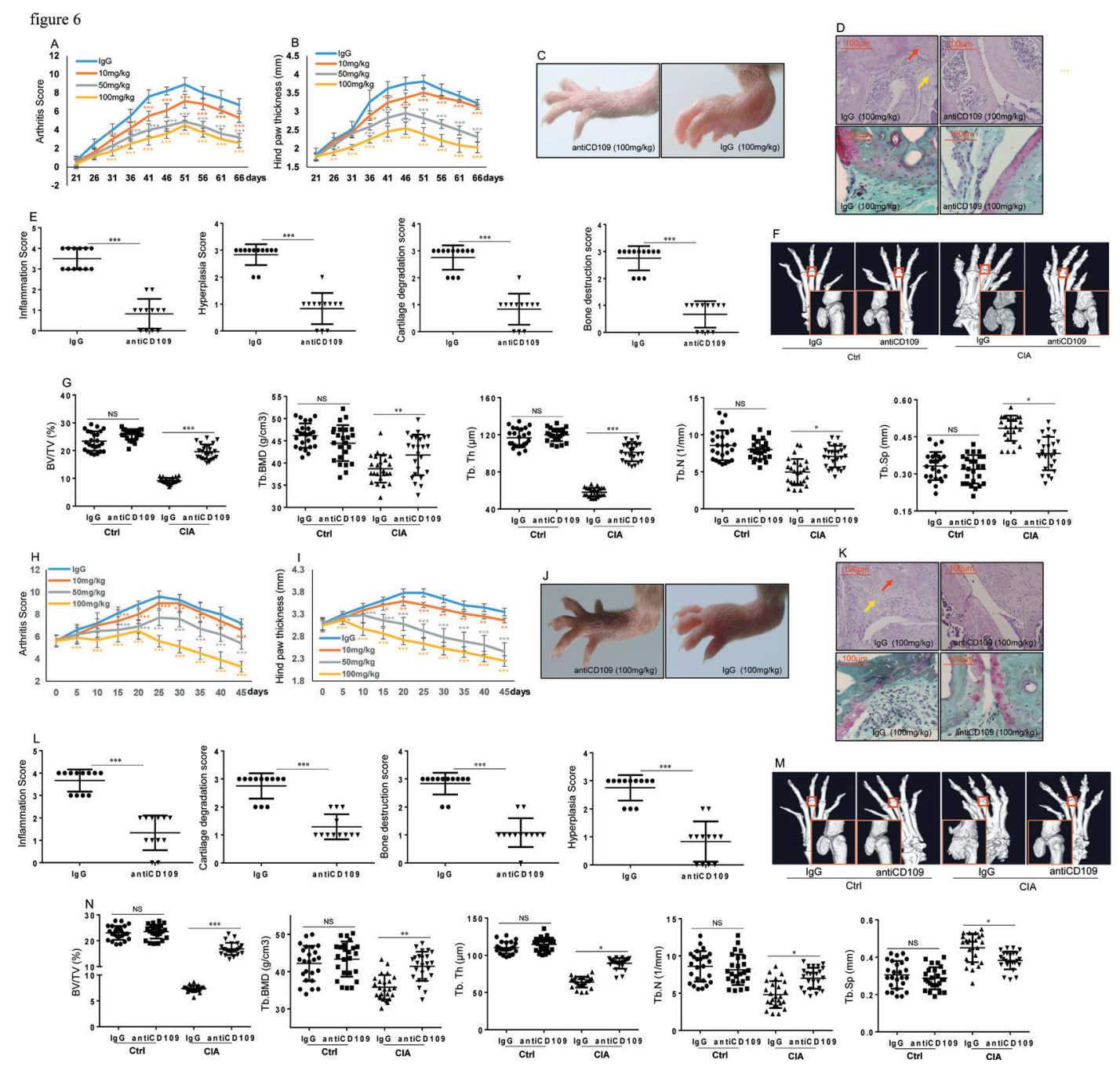

Figure 6 Antiarthritis effects of anti-CD109 on CIA. Mice immunised with CIl were randomly divided into four groups ( $n=6$ mice for each group and time point) and administered anti-CD109 or IgG at the indicated doses twice a week after the initial immunisation. The data are representative of four independent experiments with similar results. (A) Arthritis scores were monitored once per 5 days. (B) Hind paw thickness was calibrated from the 21st day following the first immunisation. (C) Paw photographs were obtained on day 42 from mice with CIA from the day of first immunisation. (D) Representative histology images by H\&E (upper) and Safranin O/ Fast green staining (lower) were obtained on day 67 from mice with CIA with the indicated treatment. Pathological changes, including synovial proliferation (yellow arrowhead) and joint destruction (red arrowhead), are shown. (E) Inflammation, hyperplasia, cartilage degradation and bone destruction were measured through a scoring system ( $n=12$ mice per group). (F) Representative micro-CT images of hind paws and interphalangeal joints (red square). (G) BV/TV, Tb BMD, Tb Th, Tb N and Tb Sp in the distal tibia were assayed by micro-CT and 3D reconstruction. Furthermore, mice immunised with CIl were divided into four groups $(\mathrm{n}=6$ mice per group and time point) equating to the mean arthritis score of individual groups. The mice were treated with anti-CD109 or lgG at the indicated doses twice a week from the point after the second immunisation when the arthritis scores reached 6 until day 45. (H) The arthritis severity was evaluated by the arthritis scores. (I) Paw swelling was measured every 5 days after anti-CD109 addition. (J) Paw photographs from mice with CIA captured on day 21 after starting anti-CD109 treatment. (K) Representative H\&E (upper) and Safranin O/Fast green staining (lower) histology images of hind paws obtained on day 46 from anti-CD109 treatment. Synovial proliferation (yellow arrowhead) and joint destruction (red arrowhead) are shown. (L) Quantification of synovitis, hyperplasia, cartilage degradation and bone destruction according to the scoring system ( $n=12$ mice per group). (M) Representative micro-CT images of hind paws and interphalangeal joints (red square). (N) BV/TV, Tb BMD, Tb Th, Tb N and Tb Sp in the distal tibia were assayed by micro-CT and $3 D$ reconstruction. $(A, B, E, H, I$ and $L){ }^{*} p<0.05,{ }^{* *} p<0.01$ and ${ }^{* * *} p<0.001$ compared with $\lg G$. $(G$ and $N){ }^{*} p<0.05$, ${ }^{* *} p<0.01$ and ${ }^{* * *} p<0.001$ compared with IgG in the Ctrl or CIA group. BMD, bone mineral density; BV/TV, bone volume fraction; CIA, collagen-induced arthritis; CII, type II collagen; NS, not significant; Tb BMD, trabecular bone mineral density; Tb N, trabecular number; Tb Sp, trabecular separation; Tb Th, trabecular thickness.

\section{DISCUSSION}

The inhibition of inflammatory reactions is an investigative focus for treating RA. ${ }^{35}$ In this study, while confirming its roles in tumour progression and tissue fibrosis, ${ }^{71236}$ we reveal a novel role for CD109 in the RA FLS-mediated immune response, such as in cytokine production, inflammatory signalling activation, migration, invasion and chemoattraction. As CD109 localises to the cell surface and exists mainly in the extracellular compartment, its activity can be effectively blocked by specific antibodies. ${ }^{37}$ Here, we found that anti-CD109 is as effective as siCD109 in attenuating the arthritis phenotype of RA FLSs. In addition, the anti-inflammatory effect of blocking CD109 in RA FLSs is equally efficient in male and female patients. More importantly, CD109 deficiency ameliorates the severity of 
arthritis in a CIA model. Anti-CD109 addition in vivo demonstrates both prophylactic and therapeutic effects in a CIA model. Together, our results suggest that CD109 may represent a novel therapeutic target for RA.

ENO1 is a classical component of the glycolytic pathway and functions in multiple ways. ${ }^{38-40}$ A previous study showed that surface ENO1 activation on monocytes and macrophages from RA patients involves intracellular p38 MAPK and NF- $\kappa$ B pathways and excessive levels of proinflammatory mediators. ${ }^{30} 32$ Furthermore, TNF- $\alpha$-induced ENO1 contributes to RA FLS IL-6 production, proliferation and survival. ${ }^{30}$ In addition, ENO1 increases plasminogen levels to enhance ECM degradation and the subsequent migration or invasion of monocytes and tumour cells. ${ }^{39}$ Here, we found that CD109 forms a complex with ENO1 and stabilises ENO1 on the cell surface, thereby mediating the inflammatory response of RA FLSs. These findings further support the hypothesis that CD109 is a crucial factor in RA pathogenesis and that ENO1 is required for CD109-mediated RA FLS activation. Further analysis would be still needed to clarify the regulatory mechanism toward the expression and activity of CD109 in RA FLSs.

CD109 deficiency caused an osteoporosis-like phenotype in vivo, while the osteoclasts seemingly matured. However, there are contradictory results from in vitro experiments. ${ }^{41}{ }^{42}$ Here, we found that CD109 loss or inhibition increased the OPG/ RANKL ratio but reduced osteoclastogenesis and bone destruction in CIA model mice, suggesting that the effect of targeting CD109 on osteoclast differentiation is context-specific and needs further clarification for the potential treatment of RA. In addition, chronic inflammation-induced bone destruction is a critical pathological feature of RA. ${ }^{43}$ RA FLSs provide the necessary signals for osteoclasts and are the main resources of RANKL that promotes osteoclast differentiation. ${ }^{34} 4546$ In this study, FLSs from CD109 KO mice were less capable than those from WT mice of supporting osteoclast differentiation in vitro, indicating that the CD109-mediated RA FLS inflammatory response towards osteoclasts also contributes to bone destruction in RA.

Based on previous studies showing that CD109 decreases excessive ECM production in systemic sclerosis fibroblasts, ${ }^{12}$ we explored whether an anti-CD109 antibody could be applied in RA patients. Our study provides a vigorous analysis of bone metabolism and potential adverse effects in organ fibrosis. CD109 is expressed in activated platelets, T cells, endothelial cells and a subpopulation of CD34-expressing cells. ${ }^{451136}$ Interestingly, these cells are important for RA progression, indicating that CD109 may contribute to RA via different routes and that the antiarthritis role of CD109 inhibition was achieved not only by RA FLSs but also by peripheral blood mononuclear cells and endothelial cells. To further benefit from CD109 as a treatment target in RA, the effects of CD109 inhibition and its underlying molecular mechanism in immune cells and endothelial cells require further investigation.

Taken together, our results uncover the proinflammatory properties of CD109. CD109 inhibition suppresses the inflammatory response and disease activity of inflammatory arthritis, and CD109 may serve as a suitable target for RA treatment.

\footnotetext{
Author affiliations

${ }^{1}$ Institute of Basic Medicine, Shandong First Medical University \& Shandong Academy of Medical Sciences, Jinan, China

${ }^{2}$ Department of Pathology, Shandong University Medical School, Jinan, China

${ }^{3}$ School of Medicine and Life Sciences, University of Jinan-Shandong Academy of Medical Sciences, Jinan, China

${ }^{4}$ Department of Hematology, Qilu Children's Hospital of Shandong University, Jinan, China
}

${ }^{5}$ Shandong Medicinal Biotechnology Centre, Key Laboratory for Rare and Uncommon Diseases of Shandong Province, Key Lab for Biotechnology Drugs of Ministry of Health, Shandong First Medical University \& Shandong Academy of Medical Sciences, Jinan, China

Acknowledgements Transgenic founder CD109+/- mice (C57Bl6:129Sv background) was designed, constructed and identified by Dr Zhang Lianfeng's laboratory at The Institute of Laboratory Animal Science, Chinese Academy of Medical Sciences. Thanks for the critical review and helpful suggestion from Dr Gao Chengjiang (Shandong University School of Medicine).

Contributors GS, LW and JH designed the research. GS, TF, QL, QG, ZW, RZ, YZ and LG performed the research. GS, YD, JP, LW and JHanalysed the data. RZ and LG helped in sample collection. All the authors contributed to writing the paper.

Funding This work was supported by the National Natural Science Foundation of China (grant nos. 81572544 and 81772760), The Shandong Taishan Scholarship (grant no. tsqn20161076) and The Innovation Project of Shandong Academy of Medical Sciences.

\section{Competing interests None.}

\section{Patient consent for publication Obtained.}

Ethics approval The studies were approved by the Institutional Review Board of Shandong Research Center for Medicinal Biotechnology.

Provenance and peer review Not commissioned; externally peer reviewed.

Data availability statement Data are available upon reasonable request. All data relevant to the study are included in the article or uploaded as supplementary information.

Open access This is an open access article distributed in accordance with the Creative Commons Attribution Non Commercial (CC BY-NC 4.0) license, which permits others to distribute, remix, adapt, build upon this work non-commercially, and license their derivative works on different terms, provided the original work is properly cited, appropriate credit is given, any changes made indicated, and the use is non-commercial. See: http://creativecommons.org/licenses/by-nc/4.0/.

\section{ORCID iD}

Lin Wang http://orcid.org/0000-0003-3489-4383

\section{REFERENCES}

1. Bottini N, Firestein GS. Duality of fibroblast-like synoviocytes in RA: passive responders and imprinted aggressors. Nat Rev Rheumatol 2013;9:24-33.10.1038/ nrrheum.2012.190

2. Bartok B, Firestein GS. Fibroblast-Like synoviocytes: key effector cells in rheumatoid arthritis. Immunol Rev 2010;233:233-55.

3. Lin M, Sutherland DR, Horsfall W, et al. Cell surface antigen CD109 is a novel member of the alpha(2) macroglobulin/C3, C4, C5 family of thioester-containing proteins. Blood 2002;99:1683-91.

4. Solomon KR, Sharma P, Chan M, et al. Cd109 represents a novel branch of the $\alpha 2$ macroglobulin/complement gene family. Gene 2004;327:171-83.

5. Hagiwara S, Murakumo Y, Mii S, et al. Processing of CD109 by furin and its role in the regulation of TGF- $\beta$ signaling. Oncogene 2010;29:2181-91.

6. Shiraki Y, Mii S, Enomoto A, et al. Significance of perivascular tumour cells defined by CD109 expression in progression of glioma. J Pathol 2017;243:468-80.

7. Hashimoto M, Ichihara M, Watanabe T, et al. Expression of CD109 in human cancer. Oncogene 2004;23:3716-20.

8. Tsai Y-L, Ha DP, Zhao H, et al. Endoplasmic reticulum stress activates Src, relocating chaperones to the cell surface where GRP78/CD109 blocks TGF- $\beta$ signaling. Proc Nat Acad Sci U S A 2018;115:E4245-E4254.

9. Yoo S-A, You S, Yoon H-J, et al. A novel pathogenic role of the ER chaperone GRP78/ BiP in rheumatoid arthritis. J Exp Med 2012;209:871-86.

10. Bizet AA, Liu K, Tran-Khanh N, et al. The TGF-beta co-receptor, CD109, promotes internalization and degradation of TGF-beta receptors. Biochim Biophys Acta 1813:2011:742-53.

11. Bizet AA, Tran-Khanh N, Saksena A, et al. CD109-mediated degradation of TGF- $\beta$ receptors and inhibition of TGF- $\beta$ responses involve regulation of Smad7 and Smurf2 localization and function. J Cell Biochem 2012;113:238-46.

12. Man X-Y, Finnson KW, Baron M, et al. Cd109, a TGF- $\beta$ co-receptor, attenuates extracellular matrix production in scleroderma skin fibroblasts. Arthritis Res Ther 2012;14.

13. Chuang $\mathrm{C}-\mathrm{H}$, Greenside $\mathrm{PG}$, Rogers $Z \mathrm{~N}$, et al. Molecular definition of a metastatic lung cancer state reveals a targetable CD109-Janus kinase-Stat axis. Nat Med 2017;23:291-300.

14. Aletaha D, Neogi T, Silman AJ, et al. 2010 rheumatoid arthritis classification criteria: an American College of Rheumatology/European League against rheumatism collaborative initiative. Arthritis Rheum 2010;62:2569-81.

15. Mclnnes IB, Schett G. Cytokines in the pathogenesis of rheumatoid arthritis. Nat Rev Immunol 2007;7:429-42. 
16. Alam J, Jantan I, Bukhari SNA. Rheumatoid arthritis: recent advances on its etiology, role of cytokines and pharmacotherapy. Biomed Pharmacother 2017;92:615-33.

17. Klein K, Kabala PA, Grabiec AM, et al. The bromodomain protein inhibitor I-BET151 suppresses expression of inflammatory genes and matrix degrading enzymes in rheumatoid arthritis synovial fibroblasts. Ann Rheum Dis 2016;75:422-9.

18. Burrage PS, Mix KS, Brinckerhoff CE. Matrix metalloproteinases: role in arthritis. Front Biosci 2006;11:529-43.

19. Li N, Xu Q, Liu Q, et al. Leonurine attenuates fibroblast-like synoviocyte-mediated synovial inflammation and joint destruction in rheumatoid arthritis. Rheumatology 2017:56:1417-27

20. Xiao $Y$, Liang $L$, Huang $M$, et al. Bromodomain and extra-terminal domain bromodomain inhibition prevents synovial inflammation via blocking $\mid \kappa B$ kinasedependent NF- $\kappa \mathrm{B}$ activation in rheumatoid fibroblast-like synoviocytes. Rheumatology 2016;55:173-84.

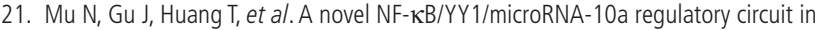
fibroblast-like synoviocytes regulates inflammation in rheumatoid arthritis. Sci Rep 2016;6:20059.

22. Tagoe CE, Marjanovic N, Park JY, et al. Annexin-1 mediates TNF- $\alpha$-stimulated matrix metalloproteinase secretion from rheumatoid arthritis synovial fibroblasts. J Immunol 2008; 181:2813-20.

23. Akhtar N, Singh AK, Ahmed S. Microrna-17 suppresses TNF- $\alpha$ signaling by interfering with TRAF2 and CIAP2 association in rheumatoid arthritis synovial fibroblasts. J.i. 2016; 197:2219-28.

24. Xu H, He Y, Yang $X$, et al. Anti-Malarial agent artesunate inhibits TNF-alpha-induced production of proinflammatory cytokines via inhibition of NF-kappaB and PI3 kinase/ Akt signal pathway in human rheumatoid arthritis fibroblast-like synoviocytes. Rheumatology 2007;46:920-6.

25. Singh AK, Fechtner $S$, Chourasia $M$, et al. Critical role of IL-1 $\alpha$ in IL-1 $\beta$-induced inflammatory responses: cooperation with NF- $\kappa B p 65$ in transcriptional regulation. The FASEB Journal 2019;33:2526-36.

26. Mori T, Miyamoto T, Yoshida H, et al. IL-1 $\beta$ and TNF $\alpha$-initiated IL-6-STAT3 pathway is critical in mediating inflammatory cytokines and RANKL expression in inflammatory arthritis. Int Immunol 2011;23:701-12.

27. Meng Q, Du X, Wang H, et al. Astragalus polysaccharides inhibits cell growth and pro-inflammatory response in IL-1 $\beta$-stimulated fibroblast-like synoviocytes by enhancement of autophagy via PI3K/Akt/mTOR inhibition. Apoptosis 2017:22:1138-46.

28. Kuan WP, Tam L-S, Wong C-K, et al. CXCl 9 and CXCL 10 as sensitive markers of disease activity in patients with rheumatoid arthritis. J Rheumatol 2010:37:257-64.

29. Ruschpler $P$, Lorenz $P$, Eichler $W$, et al. High CXCR3 expression in synovial mast cells associated with CXCL9 and CXCL10 expression in inflammatory synovial tissues of patients with rheumatoid arthritis. Arthritis Res Ther 2003;5:R241-52.
30. Bae $\mathrm{S}, \mathrm{Kim} \mathrm{H}$, Lee $\mathrm{N}$, et al. $\alpha$-Enolase expressed on the surfaces of monocytes and macrophages induces robust synovial inflammation in rheumatoid arthritis. J.i. 2012;189:365-72

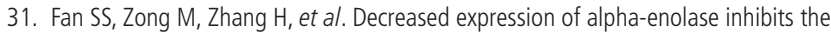
proliferation of hypoxia-induced rheumatoid arthritis fibroblasts-like synoviocytes. Mod Rheumatol 2015:25:701-7.

32 Lee JY, Kang MJ, Choi JY, et al. Apolipoprotein B binds to enolase- 1 and aggravates inflammation in rheumatoid arthritis. Ann Rheum Dis 2018;77:1480-9.

33. Choi Y, Arron JR, Townsend MJ. Promising bone-related therapeutic targets for rheumatoid arthritis. Nat Rev Rheumatol 2009:5:543-8.

34. Tanaka Y, Ohira T. Mechanisms and therapeutic targets for bone damage in rheumatoid arthritis, in particular the RANK-RANKL system. Curr Opin Pharmacol 2018;40:110-9.

35. McInnes IB, Schett G. Pathogenetic insights from the treatment of rheumatoid arthritis. The Lancet 2017;389:2328-37.

36. Winocour S, Vorstenbosch J, Trzeciak A, et al. CD109, a novel TGF- $\beta$ antagonist, decreases fibrotic responses in a hypoxic wound model. Exp Dermatol 2014;23:475-9.

37. Litvinov IV, Bizet AA, Binamer $Y$, et al. Cd109 release from the cell surface in human keratinocytes regulates TGF- $\beta$ receptor expression, TGF- $\beta$ signalling and STAT3 activation: relevance to psoriasis. Exp Dermatol 2011;20:627-32.

38. Capello M, Ferri-Borgogno S, Cappello P, et al. $\alpha$-enolase: a promising therapeutic and diagnostic tumor target. Febs j 2011;278:1064-74.

39. Wygrecka M, Marsh LM, Morty RE, et al. Enolase-1 promotes plasminogen-mediated recruitment of monocytes to the acutely inflamed lung. Blood 2009;113:5588-98.

40. Giallongo A, Feo S, Showe LC, et al. Isolation and partial characterization of a 48-kDa protein which is induced in normal lymphocytes upon mitogenic stimulation. Biochem Biophys Res Commun 1986;134:1238-44.

41. Mii S, Hoshino A, Enomoto A, et al. Cd109 deficiency induces osteopenia with an osteoporosis-like phenotype in vivo. Genes Cells 2018;23:590-8.

42. Wang $Y$, Inger $M$, Jiang $H$, et al. Cd109 plays a role in osteoclastogenesis. PLoS One 2013;8:e61213.

43. Harre U, Schett G. Cellular and molecular pathways of structural damage in rheumatoid arthritis. Semin Immunopathol 2017;39:355-63.

44. Tanaka Y, Nakayamada S, Okada Y. Osteoblasts and osteoclasts in bone remodeling and inflammation. Curr Drug Targets Inflamm Allergy 2005;4:325-8.

45. Tanaka S, Tanaka Y, Ishiguro N, et al. Rankl: a therapeutic target for bone destruction in rheumatoid arthritis. Modern Rheumatology 2018;28:9-16.

46. Danks L, Komatsu N, Guerrini MM, et al. Rankl expressed on synovial fibroblasts is primarily responsible for bone erosions during joint inflammation. Ann Rheum Dis 2016:75:1187-95. 